\title{
ARTICLE Resolvin D1 treatment on goblet cell mucin and immune responses in the chronic allergic eye disease (AED) model
}

\author{
Daniel R. Saban ${ }^{1,2}$, Robin R. Hodges ${ }^{3}$, Rose Mathew ${ }^{1}$, Nancy J. Reyes ${ }^{1}$, Chen Yu ${ }^{1}$, Rebecca Kaye ${ }^{3}$, William Swift ${ }^{3}$, Nora Botten ${ }^{3,4,5}$,
} Charles N. Serhan ${ }^{6}$ and Darlene A. Dartt ${ }^{3}$

Severe, chronic eye allergy is an understudied, vision-threatening condition. Treatments remain limited. We used a mouse model of severe allergic eye disease (AED) to determine whether topical application of the pro-resolution mediator Resolvin D1 (RvD1) terminates the response. AED was induced by injection of ovalbumin (OVA) followed by topical challenge of OVA daily. RvD1 was applied topically prior to OVA. Clinical symptoms were scored. Eye washes were assayed for MUC5AC. After 7 days, eyes were removed and the number of goblet cells, $T$ helper cell responses and presence of immune cells in draining lymph nodes and conjunctiva determined. Topical RvD1 treatment significantly reduced symptoms of AED. RvD1 did not alter the systemic type 2 immune response in the lymph nodes. AED increased the total amount of goblet cell mucin secretion, but not the number of goblet cells. RvD1 prevented this increase, but did not alter goblet cell number. Absolute numbers of CD4 $+\mathrm{T}$ cells, total CD11b + myeloid cells, eosinophils, neutrophils, and monocytes, but not macrophages increased in AED versus RvD1-treated mice. We conclude that topical application of RvD1 reduced the ocular allergic response by local actions in conjunctival immune response and a decrease in goblet cell mucin secretion.

Mucosal Immunology (2019) 12:145-153; https://doi.org/10.1038/s41385-018-0089-1

\section{INTRODUCTION}

In the face of a rising prevalence in allergic disease globally, the chronic/severe forms of allergic eye disease (AED) still remain an area of unmet medical need. These forms include atopic and vernal keratoconjunctivitis, ${ }^{1}$ and are vision-threatening owing to inflammatory-mediated scar formation of the conjunctiva and/or opacification of the cornea. ${ }^{1-4}$ Treatment regimens mostly rely on corticosteroid therapy, which is often fraught by multiple side effects, such as glaucoma, infection, and cataract. Also, there are no therapies that directly regulate excessive mucin secretion that can result in blurred vision, pain and irritation, and can lead to mucus fishing syndrome. ${ }^{5}$ Hence, a new class of treatment is needed that terminates the debilitative inflammatory responses and mucin overproduction that characterizes chronic ocular allergy.

Whereas conventional animal models of eye allergy are typically mild and self-limiting in nature, ${ }^{6}$ a more recently established system in mice, referred to as the AED model, ${ }^{7,8}$ is considered to be akin to chronic/severe forms. Disease in this model is evidenced by a robust eosinophil presence, ${ }^{9}$ blepharitis, ${ }^{10}$ conjunctival scarring ${ }^{11-13}$ thick mucoid discharge, and meibomian gland dysfunction, ${ }^{14}$ which are consistent with features of chronic disease. ${ }^{15}$ The AED model is important because the pathobiology of chronic eye allergy is understudied and renewed research efforts are needed to better understand the pathobiology and help reduce disease burden.
Both human disease and the AED model target the cornea and conjunctiva, which normally provide a barrier against the external environment. Regarding the conjunctiva, a major component of the ocular mucosa, the two principal cell types include stratified squamous and goblet cells; immune cells are also present. Each of these cell types respond to allergens and other inflammatory mediators. ${ }^{16-20}$ Conjunctival goblet cells are responsible for secretion of the mucin MUC5AC into the tear film. This mucin is high in molecular weight and as such can trap ocular allergens to remove them from the ocular surface via the lacrimal drainage system. Recent evidence demonstrates that goblet cells are direct targets of allergic mediators produced during allergy and respond to these mediators with mucin secretion. This activity is thought to be important in removal of allergens from the ocular surface and protection of the underlying epithelial cells. ${ }^{21}$ Over secretion of mucins such as MUC5AC that occurs in chronic allergy, however, can become excessive and contribute to disease. ${ }^{5}$ Secretion of goblet cell mucins in ocular allergy is mediated by their expression of all receptors for the allergic mediators histamine $(\mathrm{H} 1-\mathrm{H} 4)$ and the cysteinyl leukotrienes CysLT1 and CysLT2. ${ }^{22}$ Activation of the histamine receptors with either histamine or specific analogs for the histamine receptors or the leukotriene receptors with the leukotrienes $\mathrm{LTD}_{4}$ and $\mathrm{LTE}_{4}$ leads to secretion of high molecular weight glycoconjugates, including MUC5AC. ${ }^{22}$ Furthermore, the Th2 cytokine IL-13 can contribute to the excess mucin production by increasing goblet cell survival, mucin production, and response

\footnotetext{
'Department of Ophthalmology, Duke University School of Medicine, Durham, NC, USA; ${ }^{2}$ Department of Immunology, Duke University School of Medicine, Durham, NC, USA;

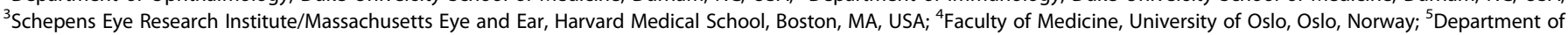
Medical Biochemistry, Oslo University Hospital, Oslo, Norway and ${ }^{6}$ Center for Experimental Therapeutics and Reperfusion Injury, Department of Anesthesiology, Perioperative and Pain Medicine, Brigham and Women's Hospital, Harvard Medical School, Boston, MA, USA

Correspondence: Darlene A. Dartt (Darlene_dartt@meei.harvard.edu)
}

Received: 9 November 2017 Revised: 17 August 2018 Accepted: 6 September 2018

Published online: 2 October 2018 
146
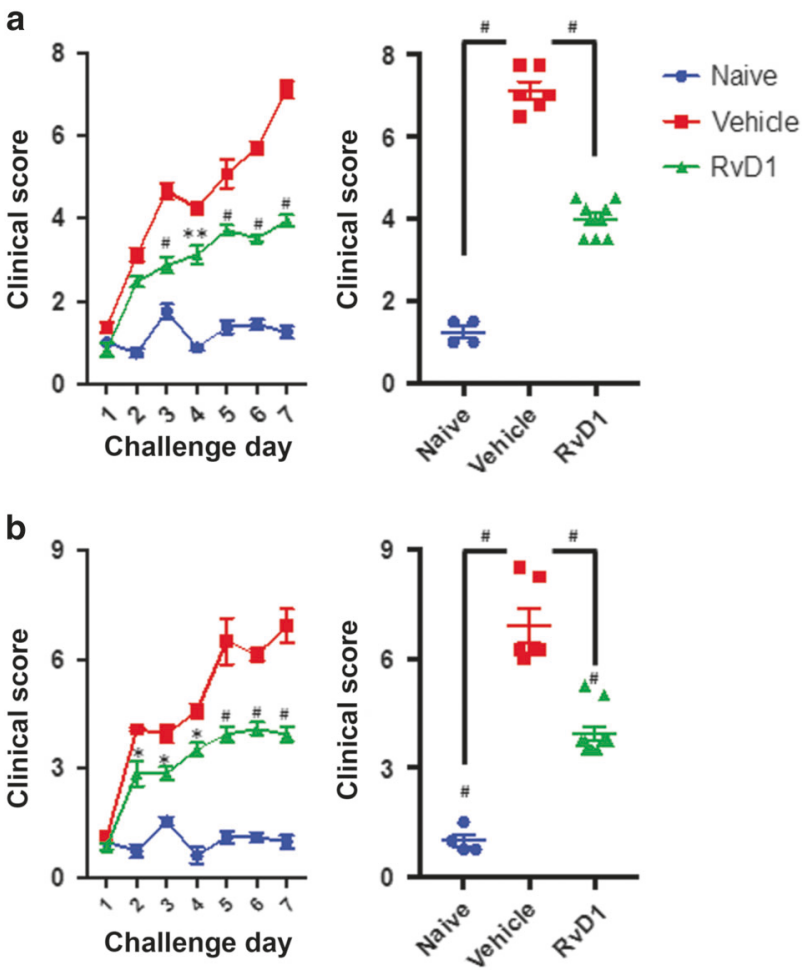

Fig. 1 AED clinical disease is significantly reduced with topical RvD1 treatment. Lid swelling, tearing, chemosis, and conjunctival redness were scored on a scale of 1-3 and summed a 20 min and $\mathbf{b} 6 \mathrm{~h}$ after topical OVA challenge. RvD1 or vehicle was added $30 \mathrm{~min}$ prior to challenge. Graphs on the right show individual mouse data points on day 7. Data are mean \pm SEM of at least four mice per condition $\left({ }^{*} p<0.05 ;{ }^{*} p<0.005 ;{ }^{\#} p<0.005\right)$. Experiment was repeated twice

to cholinergic agonists. ${ }^{17,23-25}$ Hence, overt allergic inflammatory responses and mucin over secretion are mechanistically linked and contribute to chronic disease phenotype. ${ }^{26}$

To this end, Resolvin D1 (RvD1), a specialized and endogenously produced lipid mediator in humans that actively terminates inflammation in a wide variety of tissues ${ }^{27}$ by stimulating resolution, is of particular interest and is the focus of our study herein. Resolvins (Rvs) are a family of specialized pro-resolving mediators that are derived from the omega- 3 fatty acids, i.e., eicosapentaenoic acid and docosahexaenoic acid. They are produced during the resolution phase of inflammation by resolving inflammatory exudates via a lipid mediator class switch that occurs when the production of the pro-inflammatory prostaglandins and leukotrienes changes to the biosynthesis of pro-resolving Rvs, lipoxins, and other SPMs. ${ }^{28}$ RvD1 can potentially resolve multiple types of ocular surface inflammatory processes (including mucin secretion) that are associated with chronic AED. We previously showed that conjunctival goblet cells express the receptors for RvD1 and that exogenous addition of RvD1 blocks mucin secretion stimulated by $\mathrm{LTD}_{4}, \mathrm{LTE}_{4}$, and histamine in both rat and human goblet cells. ${ }^{19,22}$ In addition, RvD1 significantly reduced angiogenic growth factor expression and infiltration of neutrophils and macrophages in a model of corneal neovascularization $^{29}$ and is not immune suppressive. Of interest, an analog of RvD1 inhibits dendritic cell maturation and reduces alloimmune responses after corneal transplantation. ${ }^{30}$ Thus, RvD1 is a potential treatment for AED with the advantage that it is endogenously present. $^{31}$

The purpose of the present study was to characterize in vivo goblet cell responses to chronic allergic mediators produced in the AED model and the role of locally administered pro-resolving mediator RvD1 in altering this response-with a particular focus on associated adaptive immune responses and disease outcomes. Our data demonstrate that topical RvD1 administration in the AED setting returned the total amount of MUC5AC in tears back to naive levels and markedly inhibited clinical disease. Interestingly, RvD1 reduced immune cell recruitment to the conjunctiva, but did not alter the systemic immune responses measured. Collectively, these results demonstrate that topical administration of RvD1 modulates otherwise overproduction of mucin secretion in the AED setting and simultaneously reduces leukocytic recruitment without affecting systemic immune responses. Hence, RvD1 could be a potential topical treatment for chronic AED without causing systemic side effects.

\section{RESULTS}

RvD1 treatment decreases clinical inflammation in the AED model We first asked whether topical RvD1 could reduce clinical reactions in an established chronic AED model. ${ }^{7,8}$ This aim was accomplished by comparing adult C57BL/6 female mice treated by a single immunization of ovalbumin (OVA)/adjuvant and subsequent topical OVA challenges (vehicle or RvD1), with mice that did not receive immunization or topical challenge (naive) (Fig. 1). These topical challenges were administered once daily for 7 consecutive days. ${ }^{7,8}$ RvD1 or vehicle was instilled topically once daily in each eye, $30 \mathrm{~min}$ prior to OVA challenge. Clinical exams were subsequently performed on a daily basis and in a masked fashion, and were conducted $20 \mathrm{~min}$ (Fig. 1a) and again at $6 \mathrm{~h}$ (Fig. 1b) post OVA challenge. ${ }^{7,8}$ The 20 min time point is consistent with immediate hypersensitivity, whereas the $6 \mathrm{~h}$ time point represents the late phase allergic reaction. ${ }^{7,8}$ Results showed a robust and statistically significant reduction in clinical scores in mice treated with RvD1, as compared with vehicle control. For the 20 min time point, reduction was seen as early as challenge day 3 (Fig. 1a), whereas reduction at the $6 \mathrm{~h}$ time point was evident beginning of day 2 (Fig. 1b). For both time points, the reduction of clinical scores continued through day 7 . Included in Fig. 1a, b (right panel) are individual data points for day 7 . Hence, these data indicate that topical RvD1 treatment significantly reduced AED at the clinical level, both for immediate hypersensitivity and late phase allergy.

RvD1 treatment does not alter systemic adaptive immunity in AED Given that secondary T helper (Th) cell responses generated in the lymph node $(L N)$ are responsible for causing $A E D,{ }^{32}$ we next wanted to ask whether the therapeutic effect seen following topical RvD1 administration is mediated by suppression of Th cell responses in the $L N$ with a particular focus on Th2. To address this question, AED was induced and mice were treated topically with RvD1 or vehicle control and compared with naive mice. On the final challenge day, LNs were harvested to assess Th cell responses via intracellular cytokine flow cytometry analysis, as previously described. ${ }^{33}$ Specifically, we assessed Th1 $(\mathrm{CD} 4+\mathrm{IFN}-\mathrm{\gamma}+)$, Th2 $(\mathrm{CD} 4+\mathrm{IL}-13+$, and IL-4 +), and Th17 (CD4 + IL-17+) cells. As expected, overall Th responses were elevated in vehicle-treated AED compared with naive mice (Fig. 2), with an exception for Th1 responses only. Intriguingly, however, RvD1 treatment of AED mice did not alter the increased cell responses compared with vehicle-treated AED mice (Fig. 2). Furthermore, we also quantified immunoglobulin $E$ ( $\mathrm{lgE}$ ) levels in the sera of these mice, as previously described. ${ }^{7}$ Raised IgE levels seen in vehicle-treated AED compared with naive mice were unchanged compared with RvD1-treated counterparts (Fig. 3). Hence, these data collectively suggest that RvD1 inhibition of AED seen at the clinical level is not due to a reduction in systemic adaptive immunity.

RvD1 treatment decreases goblet cell MUC5AC secretion in AED Our finding that RvD1 treatment in AED does not reduce Th2 responses or consequent lgE levels in circulation (and also did not 

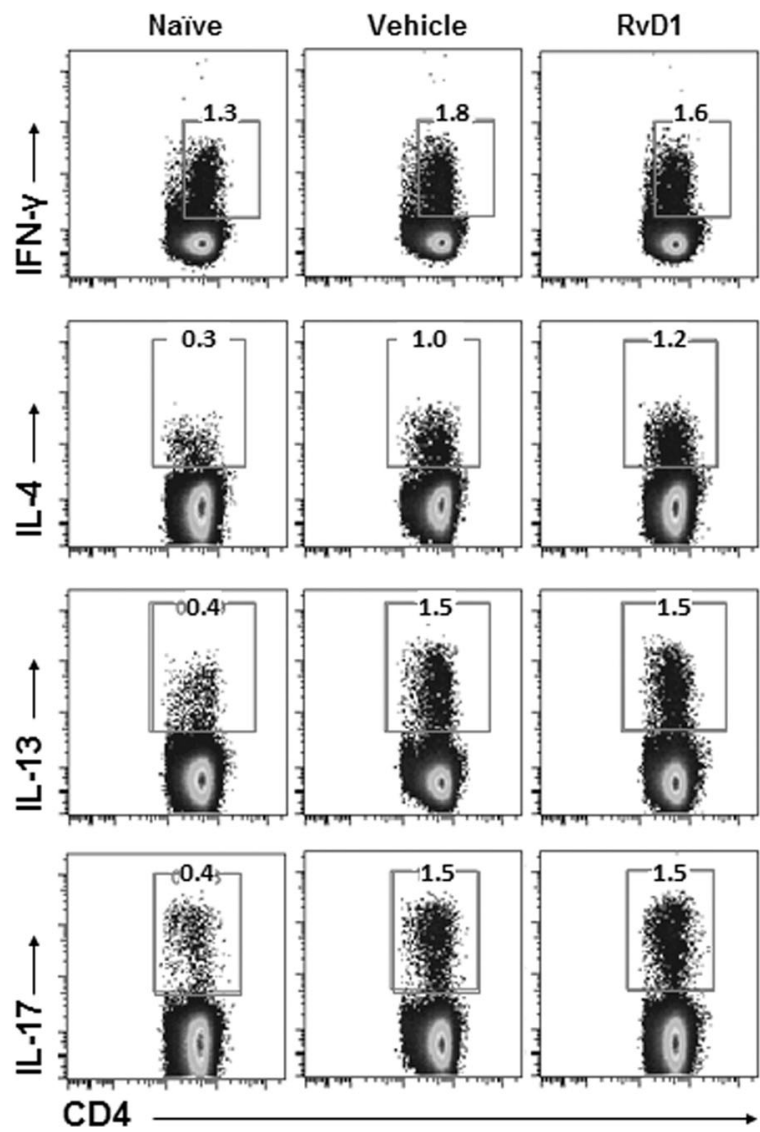

Fig. 2 T helper cell frequencies in the draining lymph node of AED mice are not affected by topical RvD1 treatment. Flow cytometry was performed on draining lymph nodes (LN) from naive, OVAtreated (vehicle), and RvD1 then OVA-treated mice for Th1 (CD4 + IFN- $\gamma+$ ), Th2 (CD4 + IL-13 + and IL-4 + ), and Th17 (CD4 + IL-17+). LNs from five mice were pooled and figure is representative of two independent experiments

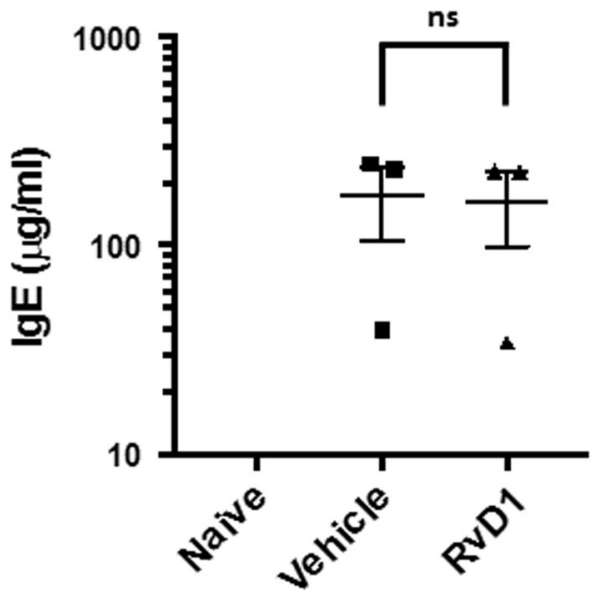

Fig. 3 Circulating lgE levels in AED mice are not affected by topical RvD1 treatment. Amount of serum IgE was measured by ELISA. Data represent three independent experiments where each experiment consisted of pooled sera from five mice for a total of 15 mice

reduce other Th responses), raised an important question regarding MUC5AC secretion (the principal mucin produced by goblet cells). Specifically, because type 2 adaptive immune responses are key in triggering goblet cell secretion, ${ }^{20,22,26}$ we wanted to ask whether RvD1 treatment in the AED setting was capable of having any inhibitory effect on MUC5AC secretion. This is an important question because overproduction of mucins in chronic eye allergy is associated with disease pathology in patients.

To address this question in the AED setting, eyes were washed with $1 \mu \mathrm{l}$ of phosphate-buffered saline (PBS) after the $20 \mathrm{~min}$ post challenge time point, on each of the 7 challenge days. Each $1 \mu \mathrm{l}$ wash was removed and assayed for MUC5AC by enzyme-linked immunosorbent assay (ELISA) and the value standardized to the tear score. We first determined if the tear score indicated an increase in tear secretion by comparing the tear score in one eye with the tear volume in the other eye as indicated by the extent of phenol red wetting in naive mice or mice in which AED was induced (untreated) (Fig. 4a). Comparison of tear score and tear phenol red thread measurement indicated a strong correlation (Spearman $r_{\mathrm{s}}=0.9288$ ). Thus tear score can be used to indicate tear volume. We next examined the tear score (which is also included in overall clinical scores shown in Fig. 1) and found in naive mice the tear score did not change over the course of the 7 days and was significantly lower than tear scores from untreated and vehicle-treated mice from days 2 to 7 . RvD1 significantly decreased tear scores compared with untreated (no topical addition before OVA treatment) and vehicle-treated (vehicle added topically before OVA treatment) mice though not to the level of naive mice (Fig. 4b). Over the course of the 7-day experiment, the amount of MUC5AC did not change in tears from naive mice (Fig. 4c) and ranged between $230.3 \pm 27.7 \mathrm{ng}$ at day 1 and $489.1 \pm 38.5 \mathrm{ng}$ at day 5 . In contrast, in the AED setting (vehicle and untreated), MUC5AC secretion increased linearly from days 1 to 4 and plateaued on days 5 to 7. The amount of MUC5AC in tears from both untreated and vehicle-treated mice was significantly increased above naive levels on all days and did not differ from each other (Fig. 4c). The MUC5AC values ranged from $303.0 \pm 21.5 \mathrm{ng}$ on day 1 to a maximum of $3242.3 \pm 161.9 \mathrm{ng}$ on day 7. Addition of RvD1 before OVA significantly decreased the amount of MUC5AC secretion as compared with untreated and vehicle-treated $A E D$ mice on all days and the values ranged from $13.2 \pm 3.0 \mathrm{ng}$ on day 1 to a maximum of $74.0 \pm 3.5 \mathrm{ng}$ on day 5 (Fig. 4c). Therefore, RvD1 decreased MUC5AC secretion in the AED setting. These results indicate that the AED setting stimulates goblet cell mucin secretion and that daily treatment with RvD1 prevents excess mucin secretion.

Conjunctival goblet cell number is unaltered in AED, irrespective of RvD1 treatment

Previous studies had shown that the number of conjunctival goblet cells decreased in a mild model of allergic conjunctivitis ${ }^{34-36}$ but recovered after final challenge. ${ }^{34}$ To interrogate whether RvD1 treatment affects goblet cell number, eyes were removed from mice $24 \mathrm{~h}$ after the end of the 7-day challenge in AED mice, fixed, and sectioned. The sections were stained with alcian blue (AB) and periodic acid/Schiff's reagent (PAS), which specifically stains the mucins present in goblet cells. The number of mucin-containing goblet cells in $6-\mu \mathrm{m}$ sections of the superior and inferior conjunctiva was then determined. As shown in Fig. $4 d$ the number of mucin-containing goblet cells in naive mice was $417.8 \pm 117.6$ cells. Intriguingly, this number was unaltered in the AED setting, and likewise in RvD1-treated AED mice. Thus goblet cell proliferation or cell death was not affected in the AED setting nor altered by RvD1 treatment.

RvD1 treatment reduces numbers of conjunctival immune cells in the AED setting

Our findings indicated that topical RvD1 therapy is proficient at preserving mucin secretion at homeostatic levels in the AED setting, even though treatment had no inhibitory effect on systemic T-cell responses. Although we have previously showed that RvD1 can directly inhibit goblet cell secretion in vitro, ${ }^{19,22}$ this 


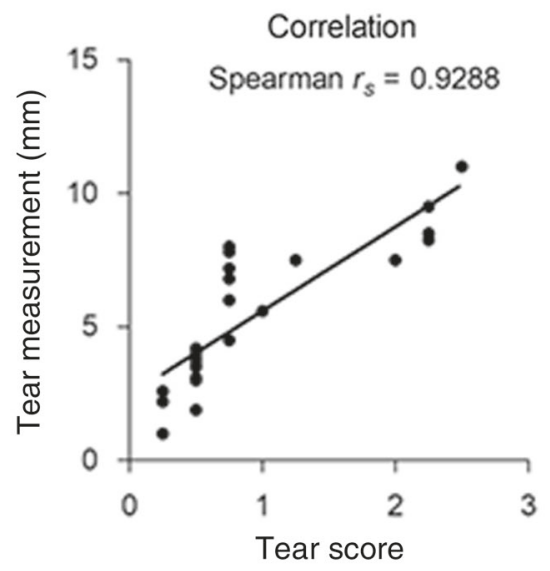

C

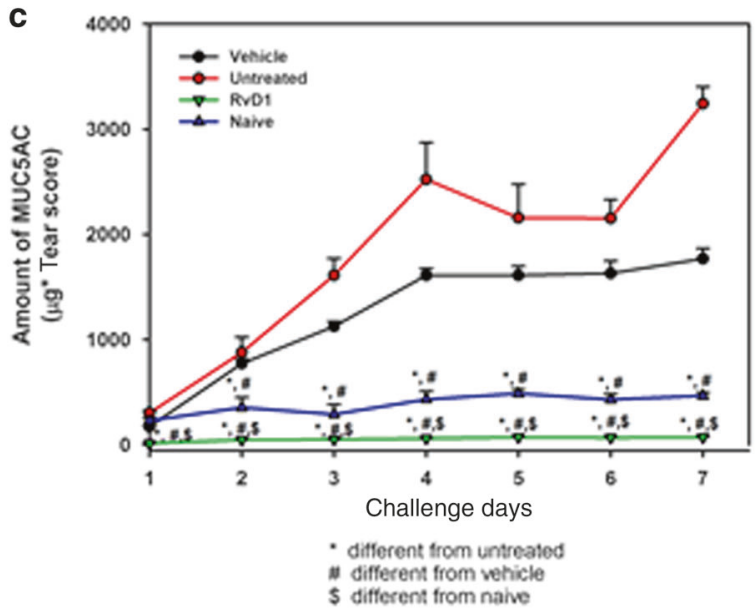

b

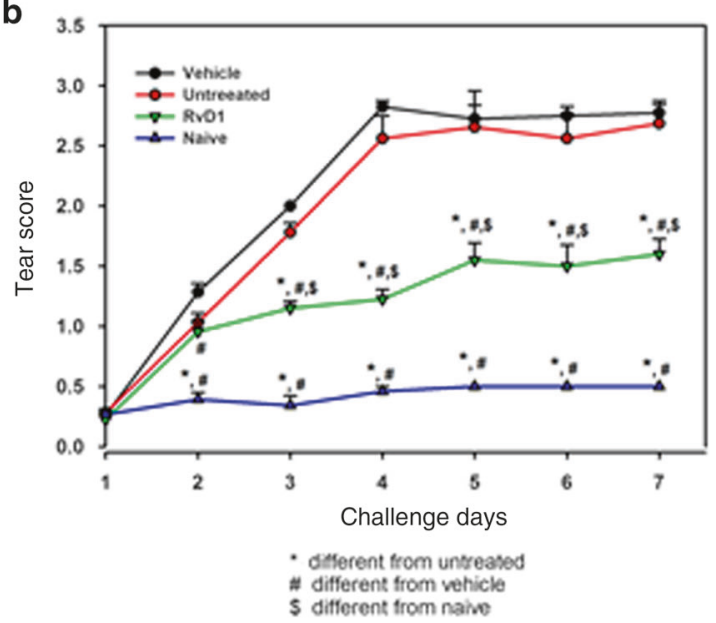

d

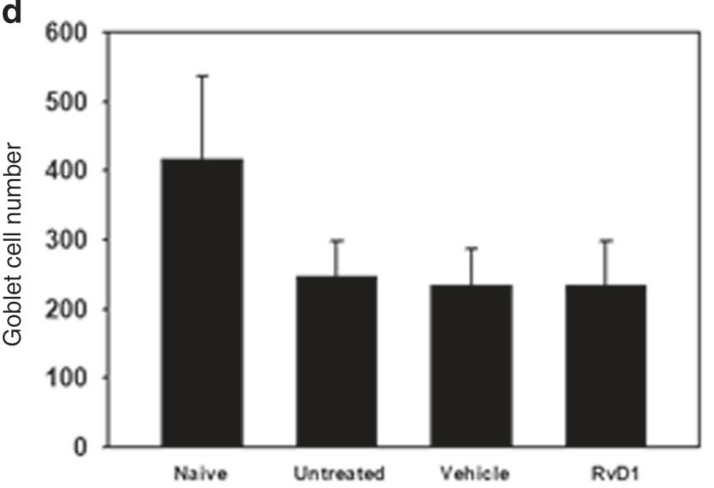

Fig. 4 Tear score and amount of MUC5AC is significantly reduced but goblet cell number was unchanged with topical RvD1 treatment. Tear amounts of naive $(n=5)$ and AED $(n=8)$ mice with different tear scores were measured using the Phenol Red Thread Test Kit in the other eye. Spearman's rank-order correlation was calculated. A best-fit line was drawn to show the trend a.Tearing was scored on a scale from 1-3 daily $20 \mathrm{~min}$ after topical application of OVA. RvD1 or vehicle was added $30 \mathrm{~min}$ prior to challenge. b Please note that tear score values are included in the overall clinical scores from Fig. 1. The amount of MUC5AC was measured in eye wash 20 min after topical challenge. Vehicle, RvD1, or no addition (untreated) was added 30 min prior to challenge. MUC5AC was measured by ELISA c. After 7 days, conjunctiva was removed, fixed, and stained with alcian blue periodic acid/Schiff's reagent. The number of goblet cells in upper and lower conjunctiva were counted d. Data are mean \pm SEM of at least 20 mice per condition. * indicates significance from untreated mice; ${ }^{\#}$ indicates significance from vehicle-treated mice; ${ }^{\$}$ indicates significance from naive mice

inhibition probably does not explain how RvD1 is able to reduce allergic inflammation at the level of the conjunctiva. Thus, in an effort to reconcile how RvD1 is able to reduce allergic inflammation, we next asked whether immune cell presence at the conjunctiva might be altered by RvD1 treatment. This aim was accomplished by harvesting conjunctivas from AED mice treated with RvD1, as compared with vehicle-treated controls. Naive samples were also collected. Tissues were prepared into single cell suspensions for multi parameter flow cytometric profiling, as previously described. ${ }^{6}$ We analyzed CD4 + T cells (CD11b-CD4+), monocytes (CD11b + Ly6C + Ly6G-), macrophages (CD11b + F4/ $80+)$, eosinophils (CD11b + Siglec $F+)$, and PMN (CD11b + Ly6G + ). In Fig. $5 a$, the respective gated populations are shown in color and overlaid onto total events (gray). To further validate the identities of these populations, we show histograms of respective identifying marker expressions of each gated population. CD4+ $\mathrm{T}$ cells uniquely express high CD4 + levels, monocytes express Ly6C, macrophages express F4/80, eosinophils express uniquely express Siglec F and neutrophils (PMN) express Ly6G (Fig. 5a).

Using this gating strategy, we analyzed the frequencies of each population in AED and whether topical treatment of RvD1 altered these frequencies. Indeed, AED showed large differences as compared with naive (Fig. 5b). T-cell frequencies in AED were increased by twofold and eosinophil frequencies were increased by fourfold. Changes in other populations were also evident (Fig. 5b). However, changes between AED vehicle-treated as compared with RvD1-treated mice were relatively marginal, as most populations were similar in both groups, e.g., T cells, eosinophils, and macrophages (Fig. 5b). Hence, it was difficult to reconcile the significant therapeutic actions of RvD1 by analyzing the differences in frequencies of these immune cell populations.

In contrast, we were able to find large differences when absolute numbers of immune cell populations were quantified in vehicle versus RvD1-treated AED mice (Fig. 5c). Specifically, CD4 + T cells, total CD11b + myeloid cells, and eosinophils had a twofold reduction with RvD1 treatment. Further, there was a fourfold reduction in PMN with RvD1 treatment, and a reduction in monocytes. In contrast, macrophages increased over twofold. These data allow us to conclude that topical RvD1 treatment reduces the absolute numbers of conjunctival immune cells in the AED setting. One exception was for macrophages that are increased, possibly to help remove dead cell debris. Thus, RvD1 
a

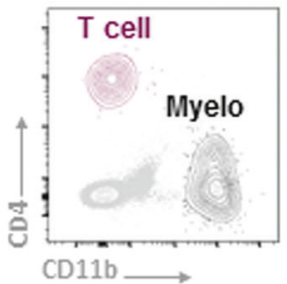

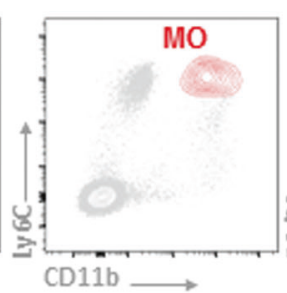

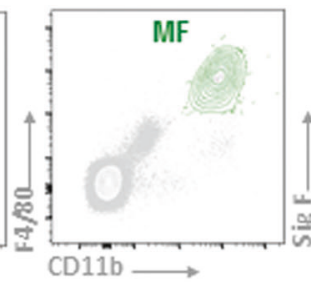

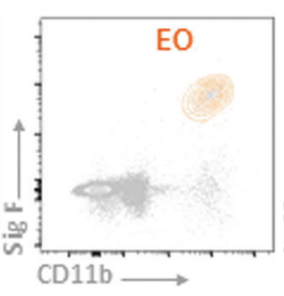
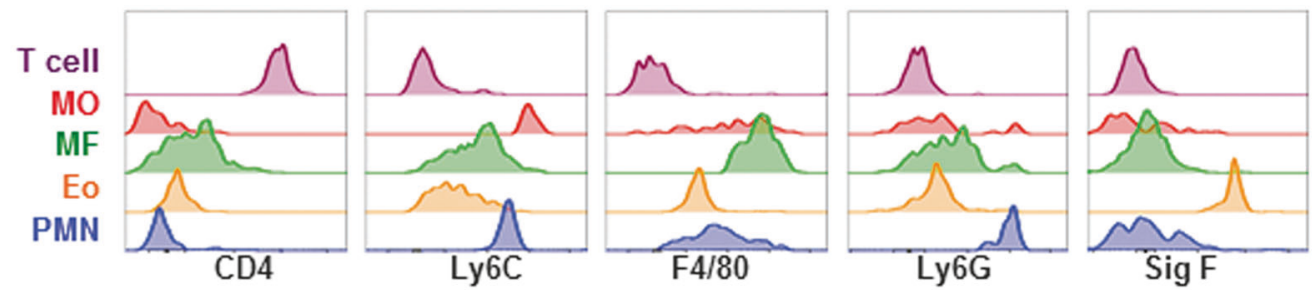

b
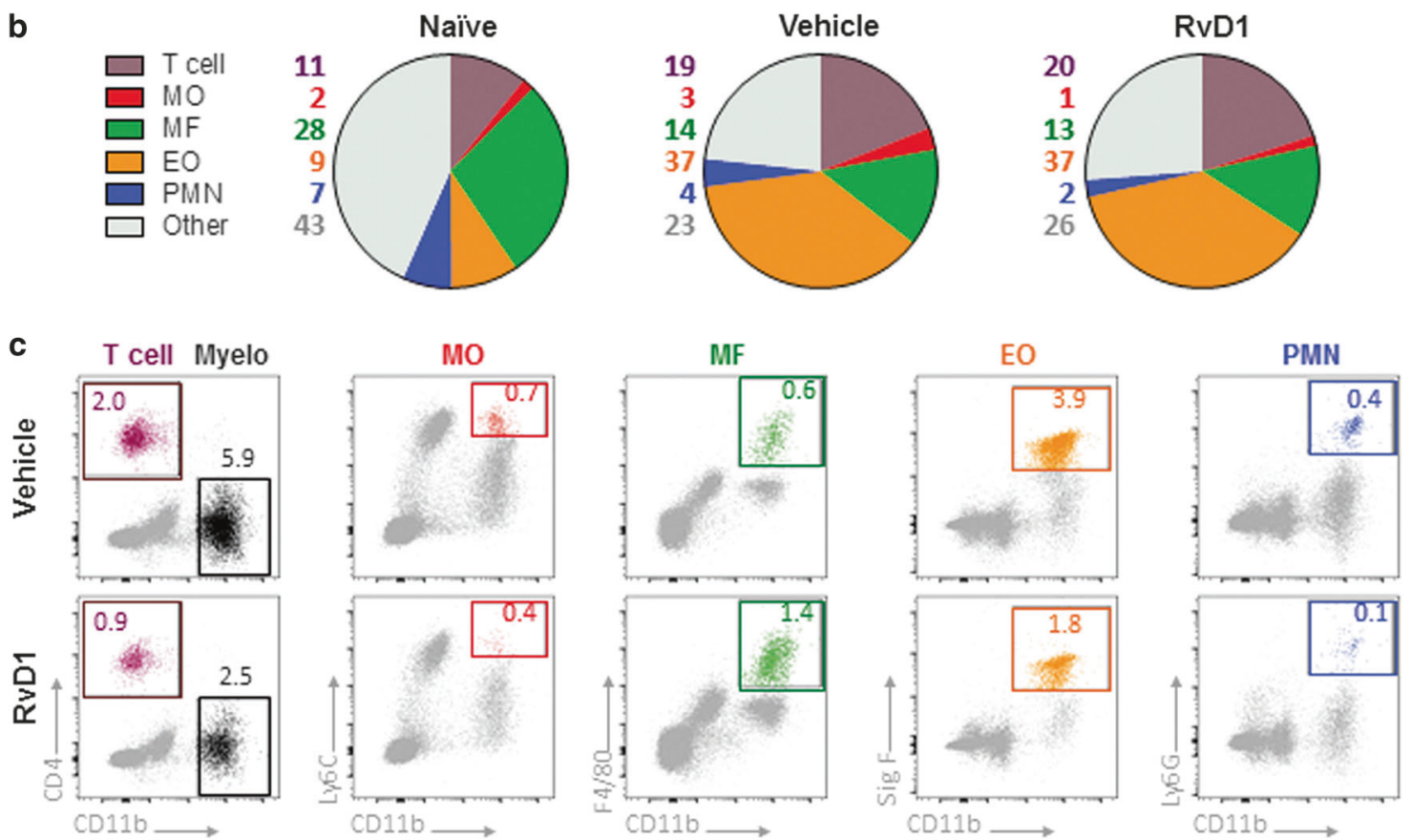

Fig. 5 RvD1 treatment reduces immune cell recruitment to the conjunctiva in AED. Flow cytometry was performed on conjunctivas from naive mice or mice treated with OVA (vehicle) or RvD1 after OVA. a Validation of respective immune cell identities in the naive conjunctiva. Top panel: Plots gated off of live singlet $C D 45+$ events in the top panel. $(\mathrm{MO}=$ monocytes; $\mathrm{MF}=$ macrophages; $E O=$ eosinophil; $\mathrm{PMN}=$ neutrophils). Bottom panel: histograms are from gated respective populations. b RvD1 treatment in AED has marginal effects on the percentages of certain immune cell populations in the conjunctiva. c RvD1 treatment results in a marked reduction of absolute numbers in CD4 + T cells and recruited myeloid cells and an increase in macrophages. Plots gated off of live, singlet, CD45 + events. Numbers in gates represent $10^{3}$ events

effectively treats AED at the local conjunctival level and is a proresolving mediator.

We also assayed for conjunctival cytokines to determine whether RvD1 modulates their levels locally. Naive mice were used as baseline, which was compared with AED mice treated with topical vehicle or RvD1. Based on our LN data, cytokines IL-4, IL-5, IL-13, IL-17A were measured. Also, as Chiurchiù et al. ${ }^{37}$ showed that RvD1 modulates IL-1 $\beta$, IL-10, and tumor necrosis factor (TNF)a levels, we additionally assayed for these cytokines as well. We used the enhanced sensitivity cytokine bead array (CBA) with subpicogram detection, thereby allowing us to ascertain accurate measurements on a per mouse basis rather than sample pooling. Of these cytokines measured, we focused on IL-1 $\beta, I L-4, I L-17 A$, and TNFa because all samples were consistently within the detectable range in all groups. Results showed that relative to naive controls, conjunctivas from AED mice showed significantly elevated levels of IL-4, IL-17A, and TNFa (Fig. 6). There are no significant changes in IL-1 $\beta$ under the three conditions. Interestingly, however, RvD1-treated AED conjunctivas did not have elevated levels IL-4 or TNFa levels relative to naive controls. Also, IL-17A levels in the RvD1 group trended closer to the naive controls than did the vehicle control. Thus, our results indicate that RvD1 does significantly modulate IL-4 and TNFa levels at the conjunctival level.

\section{DISCUSSION}

We herein show that treatment with RvD1 has a robust therapeutic effect in the AED model including a decrease in clinical symptoms, tear MUC5AC amount (without a change in the 

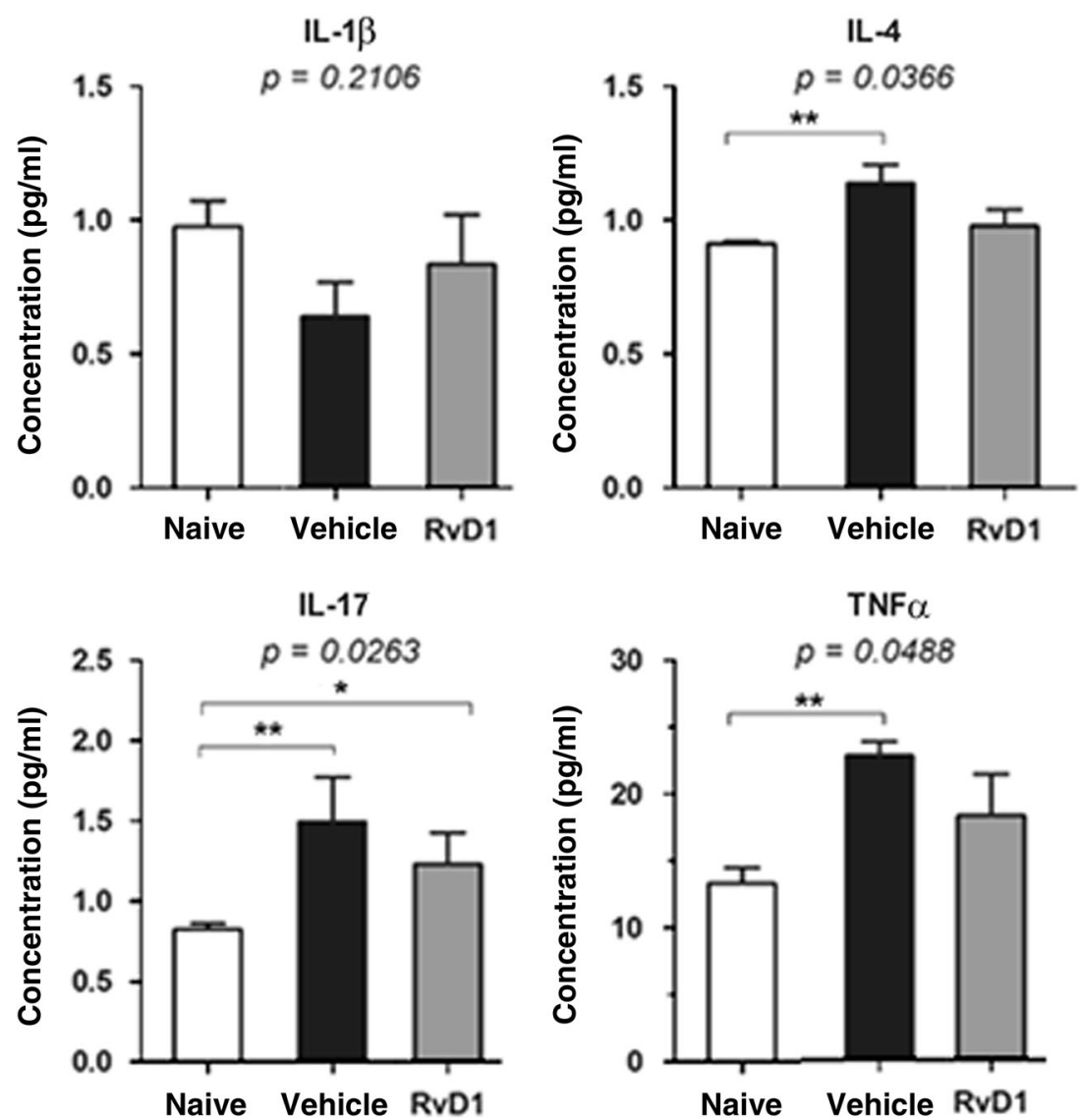

Fig. 6 Characterization of RvD1 treatment on conjunctival cytokine levels in AED. Multiplex cytokine analysis was performed on protein isolates from the conjunctivas of naive mice and AED mice treated with RvD1 or vehicle control. Data are expressed as means \pm SEMs from unpooled $n=4$ mice/group) and experiment was repeated three times (Kruskal-Wallis test with Conover-Inman post hoc test)

number of mucin-containing goblet cells), the number of immune cells recruited to the conjunctiva (except for macrophages), and modulation of certain cytokines in the conjunctiva. All of these responses suggest a dampening of the allergic inflammatory response that is consistent with the known pro-resolution mechanism of action of RvD1. ${ }^{38}$ Our findings are important and potentially relevant because chronic AED is an area of unmet medical need, and thus topical RvD1 administration may hold promise to this end.

In the present study, RvD1 was applied topically to the ocular surface, which is in contrast to animal models of other inflammatory diseases in which RvD1 was given i.v. This advantage allowed us to investigate the systemic versus local effect of RvD1. In so doing, we found that the immune actions of RvD1 were not systemic, but rather local action in the conjunctiva decreasing the number of immune cells and levels of certain cytokines. Interestingly, although type 2 immune responses are the central pathogenic mediators of allergy (including in $A E D^{32}$ ), we could not detect a change in Th2 frequencies at the level of the LN. Likewise, we showed that IgE levels in the serum were unaffected in RvD1-treated AED mice as well, which is consistent with unchanged Th2 responses in the LN. Indeed, Th2 is central to B-cell activity and consequent IgE production. Other Th cell frequencies, including Th1 and Th17, were also only marginally altered by RvD1 treatment in AED. Hence, taken together we can conclude that topical RvD1 treatment in AED mice does not alter systemic immune responses. This unaltered state can be explained by the knowledge that topical treatment does not affect the lymphoid organs, as only trace amounts of topical drugs in the eye are typically found in systemic circulation. ${ }^{39}$ In contrast, earlier we showed that a topical CCR7 antagonist does in fact affect LN responses; however the mechanism is through inhibition of conjunctival dendritic cell migration to the local LN. ${ }^{33,40}$ The local action of topical RvD1 treatment could decrease its side effects compared with other topical treatments and RvD1 given systemically and could be an advantage as a potential therapy.

Further to the subject of immune cells, we were able to find a reduction in absolute numbers of leukocytes and lymphocytes in the conjunctiva. Whereas the frequency of each immune cell population was relatively similar in RvD1 treatment vs. vehicle control AED mice (with some exception with neutrophils and monocytes), the absolute numbers were substantially lowered in the treatment group. These results suggest that RvD1 does not alter the immune response profile, but rather it causes an overall dampening of total the response locally, potentially through local inhibition of recruitment. However, we did find an unique increase in the absolute numbers of macrophages from the conjunctiva of RvD1-treated AED animals compared with vehicle. These results may suggest that macrophages were still present to remove apoptotic PMNs, cellular debris, and other immune cells that would otherwise perpetuate the inflammatory response. The latter is a hallmark of a pro-resolving phenotype. We also found that RvD1 modulates the levels of IL-4 and TNFa at the conjunctival level, further demonstrating the local tissue effect of topical administration.

The increase in tear volume is a well-known symptom/sign of ocular allergy and could result from an increase in lacrimal gland and conjunctival epithelial cell electrolyte and water secretion, as well as an increase in conjunctival blood vessel permeability. This AED manifestation is stimulated by histamine and other allergic mediators such as leukotrienes. ${ }^{41,42}$ There are, however, no studies on the impact of pro-resolving mediators on the 
stimulation of lacrimal gland and conjunctival fluid secretion or on tear secretion.

Along with the increase in tear volume is an increase in the total MUC5AC amount in the tears. This goblet cell-specific mucin is gel forming and thought to trap allergens to aid in their clearance ${ }^{21}$; its overproduction has been shown in several allergic conditions. $^{43,44}$ The amount of MUC5AC is regulated by controlling its synthesis, and secretion, as well as the number of goblet cells that contain mucin. ${ }^{45}$ In asthma, mucin overproduction and hypersecretion of MUC5AC is well described and the number of MUC5AC containing goblet cells increases dramatically. ${ }^{46}$ Although increased mucin secretion has been observed in mild allergic conjunctivitis models, ${ }^{47}$ the question of MUC5AC levels specifically has not been addressed, nor was this factor looked at in a chronic disease setting, as recapitulated in AED mice. In another study using a model of mild allergic conjunctivitis, MUC5AC mRNA was reported to be decreased immediately after challenge, but recovered by $6 \mathrm{~h}$ and was unchanged at any time when challenged with the peptide P3-1 (a product of cat dander). ${ }^{34}$ Data presented in our study demonstrate that MUC5AC secretion, as measured by ELISA, is increased in the AED model, which is inhibited by RvD1. The difference in the two studies could be explained by the possibility that a change in MUC5AC synthesis is not correlated to its secretion. MUC5AC synthesis is a long-term process controlled by EGF in airway and nasal epithelium. ${ }^{48,49}$ In contrast MUC5AC secretion is a rapid process regulated by neural and autacoid stimulation of secretory granule fusion and release of MUC5AC and other granule constituents. ${ }^{19,50,51}$ In vivo neural stimulation of secretion occurred by $5 \mathrm{~min}$, the shortest time measured.

The mechanism by which RvD1 exerts its effects on conjunctiva to attenuate the symptoms of AED is not understood as there are multiple potential cell targets. In addition to goblet cells, stratified squamous cells in the conjunctiva also express ALX/FPR2, the receptor to which RvD1 binds. ${ }^{52}$ Infiltrating neutrophils also express ALX/FPR2 and its expression was increased in endothelial cells under stress conditions. ${ }^{53}$ In the same study, RvD1 reduced the recruitment of neutrophils. ${ }^{53}$ In the setting of AED, treatment with RvD1 decreases the absolute number of neutrophils present in the conjunctiva. Thus neutrophils along with goblet cells could be targets of RvD1.

Despite an increase in amount of MUC5AC secretion seen in AED mice, we did not detect a change in the number of conjunctival goblet cells in these experiments between any of the conditions. Kunert et al. ${ }^{34}$ observed a decrease in the number of filled goblet cells immediately after and until $48 \mathrm{~h}$ after final challenge in their model of mild allergy. This discrepancy could be due to several reasons. In the present study, the eyes were removed $24 \mathrm{~h}$ after the final challenge, so it is possible that there were differences in goblet cell numbers early in the study but that stabilized within the $24 \mathrm{~h}$ after the 7 day challenge. Filled goblet cells were identified using actin-binding phalloidin in the Kunert study, whereas in the current study AB/PAS was used, which directly stains the mucins in filled goblet cells. ${ }^{34}$ Finally, Kunert et al. used whole mounts of the conjunctiva and counted goblet cells in the fornix only. In contrast, in the current study, goblet cells were counted in sections from the entire length of the conjunctiva including the superior and inferior bulbar, forniceal (fornix), and tarsal conjunctiva.

The finding that goblet cell MUC5AC secretion is increased in our AED model and that RvD1 decreases this secretion toward control levels is consistent with our published work on cultured human and rat conjunctival goblet cells. Receptors for RvD1 are expressed on both stratified squamous epithelial and goblet cells. ${ }^{18,22}$ RvD1 blocked glycoconjugate secretion from cultured goblet cells stimulated by the leukotriene $\mathrm{LTD}_{4}$ and histamine, both of which are major mediators of AED. ${ }^{16,54}$ In addition,
RvD1 stimulated glycoconjugate secretion on its own implying that RvD1 has a role in goblet cell function under physiologic conditions. There are no studies regarding the effects of RvD1 on conjunctival stratified squamous cells, but RvD1 could be effective on these cells as the DRV1(GPR32) receptor is present. ${ }^{19}$

We conclude that topical RvD1 decreases clinical signs, goblet cell mucin secretion, and conjunctival recruitment of immune cells that all together resolve the allergic inflammatory response. The action of RvD1 was local, on the conjunctiva, but not the draining lymph nodes, suggesting that topical RvD1 could be an effective treatment of chronic/severe forms of ocular allergy and that it would have the benefit of reducing unwanted systemic side effects.

\section{MATERIALS AND METHODS}

Animals and euthanasia

Eight-week-old C57BL/6 mice were purchased from Charles River Laboratories (Wilmington, MA, USA). Mice were housed at the Duke University Eye Research Institute Animal Facility (Durham, NC, USA). Euthanasia was performed according to the American Veterinary Medical Association Guidelines for Euthanasia of Animals: $\mathrm{CO}_{2}$ asphyxiation, followed by bilateral thoracotomy to ensure non-recovery. The Institutional Animal Care and Use Committee approved all procedures, and all animals were treated according to guidelines established by the National Institutes of Health Guide for the Use of Laboratory Animals.

Induction of ocular allergy by active immunization

As previously described, $7,8,33$ mice were immunized with an intraperitoneal injection of $10 \mathrm{mg}$ OVA (Sigma-Aldrich, St. Louis, $\mathrm{MO}$, USA) in $300 \mathrm{ng}$ pertussis toxin (Sigma-Aldrich) and $1 \mathrm{mg}$ aluminum hydroxide (Thermo Fisher Scientific, Waltham, MA, USA) in PBS. After a 14 day rest period, mice were given topical application of $250 \mathrm{mg}$ OVA solution (i.e., OVA challenged) once/ day to each eye for at least 7 days. Naive mice did not receive the OVA challenge.

\section{Treatment with RvD1}

Thirty minutes prior to OVA challenge, $10 \mathrm{ng}$ of RvD1 per eye for a total of $20 \mathrm{ng}$ RvD1 (7S,8 R,17S-trihydroxy-docosa$4 Z, 9 E, 11 E, 13 Z, 15 E, 19 Z$-hexaenoic acid) ${ }^{55,56}$ in ethanol (as supplied by Cayman Chemicals, Ann Arbor, MI) diluted with PBS giving 2\% ethanol (denoted as RvD1 in the figures) or vehicle (PBS containing $2 \%$ ethanol (termed vehicle in the figure)) were topically administered. This amount of RvD1 was recently shown to be present in human emotional tears. ${ }^{31}$

Clinical scoring of AED

The allergic response was scored as previously described. ${ }^{6,10,15}$ In brief, each of the following parameters-lid swelling, tearing, chemosis, and conjunctival redness-were scored on a scale of $1-3$ and summed. ${ }^{57}$ Scoring was done twice/day, at 20 min and at $6 \mathrm{~h}$ after challenge to assess the immediate hypersensitivity and late phase reaction, respectively.

\section{Tear measurement for comparison with tear scoring}

Tear amounts were assessed in 13 naive or AED mice by using the Phenol Red Thread Test Kit. All mice were challenged, and scored $20 \mathrm{~min}$ later. Immediately after $20 \mathrm{~min}$ scoring, mice were examined for tear measurements. For tear measurement cotton threads were placed in the conjunctival fornix for $30 \mathrm{~s}$. The length of wet thread was measured in $\mathrm{mm}$. Of 13 mice evaluated one eye was excluded due to excess mucin discharge as excess mucin is not absorbed by the cotton thread. Alternate eyes from each mouse was used for tear measurement. Each dot on graph represents tear score and tear measurement from the same eye. 


\section{Measurement of MUC5AC secretion}

Each mouse eye was washed with $1 \mu$ of sterile saline 30 min after addition of RvD1 followed by OVA for 20 min. The $1 \mu \mathrm{l}$ wash was collected immediately and stored at $-20^{\circ} \mathrm{C}$. Samples were diluted 20 -fold with PBS (145 mM NaCl, $7.3 \mathrm{mM} \mathrm{Na}_{2} \mathrm{HPO}_{4}$, and $2.7 \mathrm{mM}$ $\mathrm{NaH}_{2} \mathrm{PO}_{4}(\mathrm{pH}$ 7.2)) and the amount of MUC5AC in each sample was determined in duplicate using MUC5AC ELISA kit (Biotang, Lexington, $M A$ ) according to manufacturer's instructions. Amount of MUC5AC was corrected for the amount of tearing as a dilution factor by multiplying by the tear score as determined by scoring each mouse on a scale of $0-3+$.

Quantification of number of mucin-containing goblet cells Eyes from mice were removed at the end of the challenge period, fixed in $4 \%$ formaldehyde, and embedded in paraffin. Six micron sections were cut and stained with AB/PAS. The number of goblet cells in both upper and lower conjunctiva was counted in a blind fashion in three sequential sections and values averaged. This technique identifies goblet cells based on their mucin content. Goblet cells that have secreted and released their contents would not be counted.

Digestion of conjunctiva

As described previously, ${ }^{33}$ conjunctival tissue was collected after the final day of challenge and placed in digestion buffer consisting of Hank's balanced salt solution with $0.2 \%$ collagenase D (Roche Diagnostics, Indianapolis, IN, USA) and $0.01 \%$ DNase (Roche Diagnostics). Samples were placed in a $37^{\circ} \mathrm{C}$ water bath and subjected to quick dissociation by vortexing at a moderate speed at $15-20$ min intervals over a $1 \mathrm{~h}$ period. Ethylenediaminetetraacetic acid disodium salt solution $(75 \mathrm{ml}$; Sigma-Aldrich) was added to stop the digestion reaction.

\section{LN helper cell analysis}

This procedure was performed as described previously. ${ }^{8}$ In brief, on the last day of challenge, draining LNs were collected from freshly killed mice and processed. Single cell suspensions were prepared and enumerated via trypan blue exclusion assay. Cells were resuspended in Roswell Park Memorial Institute medium (10\% fetal bovine serum) and plated at a concentration of $2 \times 10^{6}$ cells $/ \mathrm{ml}$ in round bottom $96-$ well plates. Cells were stimulated for $4 \mathrm{~h}$ with $0.2 \%$ phorbol myristate acetate + ionomycin + Golgi block (eBioscience) for flow analysis.

Flow cytometry and analysis

For conjunctival staining, the following antibodies or dyes were used for flow cytometry: Viability (65-0863-14, eBioscience), F4/80 (BM8, eBioscience), Ly6C (HK1.4, BioLegend), Ly6G (1A8, BioLegend), CD64 (X54-5/7.1, BioLegend), I-A/I-E (M5/114.15.2, BioLegend), CD11b (M1/70, BD Pharmingen), CD11C (HL3, BD Pharmingen), CD45 (30-F11, BioLegend), Siglec F (E50-2440, BD Pharmingen) CD4 (RM4-5, BD Pharmingen). For dLN cells: viability, extracellular CD4 (RM4-5, BD Pharmingen) and intracellular IL-4 (11B11, BioLegend), IL-13 (eBio13A, eBioscience), IL-17(TC11$18 \mathrm{H} 10, \mathrm{BD}$ Pharmingen), and IFN- $\gamma$ (XMG1.2, Biolegend). Data were acquired on BD Fortessa LSRII and analyzed on FloJo (Treestar Inc.)

\section{$\lg$ E ELISA}

Following OVA challenge on day 7 , blood was collected from mice via cardiac puncture and sera was isolated. Samples were analyzed via ELISA for total serum $\mathrm{lgE}$ according to the manufacturer's instructions (eBioscience).

\section{Cytokine bead array}

Conjunctival tissue was collected at day 7 of treatment. Proteins were extracted in $120 \mu \mathrm{l}$ RIPA buffer supplemented with PMSF and proteinase inhibitor cocktails. Concentrations of cytokines, including IL-1 $\beta$, IL-4, IL-5, IL-10, IL-13, IL-17A, and TNFa, were measured using BD CBA Mouse Enhanced Sensitivity Kit and analyzed using FlowJo and Graphpad prism. The readout numbers of IL-5, IL-10, and IL-13 were lower than the lowest standards $(274 \mathrm{fg} / \mathrm{ml})$, and thus they were not included in analysis.

\section{Statistical analysis}

Experiments were done using groups of five mice and repeated at least twice. Data are presented as mean \pm SEM. Data were analyzed by analysis of variance (ANOVA) followed by Tukey post hoc test or Student's $t$ test. $P<0.05$ was considered statistically significant. For tear correlation studies, Spearman's rank-order correlation was calculated and a best-fit line was drawn to show the trend. For cytokine bead array analysis, data normality and homogeneity of variance were assessed using Shapiro-Wilk test and Levene's test, respectively. As either normality or homogeneity of variance did not fit the assumptions of one-way ANOVA, non-parametric statistical analyses of Kruskal-Wallis test with Conover-Inman post hoc test was used.

\section{ACKNOWLEDGEMENTS}

We thank Dr. Tomas Blanco-Mezquita. This study was funded by NIH R01 EY019470 (DAD), R01EY021798 (DRS), P30EY005722 (Duke University), P01GM095467 (CNS), and P30EY003790 (Schepens Eye Research Institute). Supported by NIH RO1 EY019470 (Dartt), P30EY005722 (Saban), R01EY021798 (Saban)

\section{AUTHOR CONTRIBUTIONS}

DRS-designed experiments, analyzed results, wrote manuscript; RRH-performed experiments, analyzed results, wrote manuscript; RM-performed experiments, analyzed results, wrote manuscript; NJR-performed experiments; CY-performed experiments; RK-analyzed results; WS-performed experiments; NB-performed experiments; CNS-designed experiments and wrote manuscript; DAD-designed experiments, analyzed results, wrote manuscript.

\section{ADDITIONAL INFORMATION}

Competing interests: The authors declare no competing interests.

\section{REFERENCES}

1. Gomes, P. J. Trends in prevalence and treatment of ocular allergy. Curr. Opin. Allergy Clin. Immunol. 14, 451-456 (2014).

2. O'Brien, T. P. Allergic conjunctivitis: an update on diagnosis and management. Curr. Opin. Allergy Clin. Immunol. 13, 543-549 (2013).

3. Dale, S. B. \& Saban, D. R. Linking immune responses with fibrosis in allergic eye disease. Curr. Opin. Allergy Clin. Immunol. 15, 467-475 (2015).

4. Lee, H. S. et al. Involvement of corneal lymphangiogenesis in a mouse model of allergic eye disease. Invest. Ophthalmol. Vis. Sci. 56, 3140-3148 (2015).

5. McCulley, J. P., Moore, M. B. \& Matoba, A. Y. Mucus fishing syndrome. Ophthalmology 92, 1262-1265 (1985).

6. Groneberg, D. A., Bielory, L., Fischer, A., Bonini, S. \& Wahn, U. Animal models of allergic and inflammatory conjunctivitis. Allergy 58, 1101-1113 (2003).

7. Lee, H. S., Schlereth, S., Khandelwal, P. \& Saban, D. R. Ocular allergy modulation to hi-dose antigen sensitization is a Treg-dependent process. PLOS. ONE 8, e75769 (2013).

8. Khandelwal, P. et al. Ocular mucosal CD11b+and CD103+mouse dendritic cells under normal conditions and in allergic immune responses. PLOS. ONE 8, e64193 (2013).

9. Reyes, N. J. \& Saban, D. R. T helper subsets in allergic eye disease. Curr. Opin. Allergy Clin. Immunol. 14, 477-484 (2014).

10. Kari, O. \& Saari, K. M. Diagnostics and new developments in the treatment of ocular allergies. Curr. Allergy Asthma Rep. 12, 232-239 (2012).

11. Ahadome, S. D. et al. Aldehyde dehydrogenase inhibition blocks mucosal fibrosis in human and mouse ocular scarring. JCl Insight 1, e87001 (2016).

12. Ahadome, S. D. et al. Classical dendritic cells mediate fibrosis directly via the retinoic acid pathway in severe eye allergy. JCI Insight 1, e87012 (2016).

13. Leonardi, A., Curnow, S. J., Zhan, H. \& Calder, V. L. Multiple cytokines in human tear specimens in seasonal and chronic allergic eye disease and in conjunctival fibroblast cultures. Clin. Exp. Allergy 36, 777-784 (2006). 
14. Reyes, N. J., Mathew, R. \& Saban, D. R. Induction and characterization of the allergic eye disease mouse model. Methods Mol. Biol. 1799, 49-57 (2018).

15. Solomon, A. Corneal complications of vernal keratoconjunctivitis. Curr. Opin. Allergy Clin. Immunol. 15, 489-494 (2015).

16. Garcia-Posadas, L. et al. Interaction of IFN-gamma with cholinergic agonists to modulate rat and human goblet cell function. Mucosal Immunol. 9, 206-217 (2016).

17. Garcia-Posadas, L., Contreras-Ruiz, L., Soriano-Romani, L., Dartt, D. A. \& Diebold, Y. Conjunctival goblet cell function: effect of contact lens wear and cytokines. Eye Contact Lens 42, 83-90 (2016).

18. Hodges, R. R., Li, D., Shatos, M. A., Serhan, C. N. \& Dartt, D. A. Lipoxin A4 counterregulates histamine-stimulated glycoconjugate secretion in conjunctival goblet cells. Sci. Rep. 6, 36124 (2016).

19. Li, D. et al. Resolvin D1 and aspirin-triggered resolvin D1 regulate histaminestimulated conjunctival goblet cell secretion. Mucosal Immunol. 6, 1119-1130 (2013).

20. Hayashi, D. et al. Role of histamine and its receptor subtypes in stimulation of conjunctival goblet cell secretion. Invest. Ophthalmol. Vis. Sci. 53, 2993-3003 (2012).

21. Mantelli, F. \& Argueso, P. Functions of ocular surface mucins in health and disease. Curr. Opin. Allergy Clin. Immunol. 8, 477-483 (2008).

22. Dartt, D. A. et al. Conjunctival goblet cell secretion stimulated by leukotrienes is reduced by resolvins $\mathrm{D} 1$ and $\mathrm{E} 1$ to promote resolution of inflammation. $J$. Immunol. 186, 4455-4466 (2011).

23. Contreras-Ruiz, L., Ghosh-Mitra, A., Shatos, M. A., Dartt, D. A. \& Masli, S. Modulation of conjunctival goblet cell function by inflammatory cytokines. Mediators. Inflamm. 2013, 636812 (2013).

24. De Paiva, C. S. et al. Homeostatic control of conjunctival mucosal goblet cells by NKT-derived IL-13. Mucosal Immunol. 4, 397-408 (2011).

25. Tukler Henriksson, J., Coursey, T. G., Corry, D. B., De Paiva, C. S. \& Pflugfelder, S. C. IL-13 stimulates proliferation and expression of mucin and immunomodulatory genes in cultured conjunctival goblet cells. Invest. Ophthalmol. Vis. Sci. 56, 4186-4197 (2015).

26. Garcia-Posadas, L., Hodges, R. R., Diebold, Y. \& Dartt, D. A. Context-dependent regulation of conjunctival function by allergic mediators. Sci. Rep. 8, 12162 (2018).

27. Serhan, C. N. Discovery of specialized pro-resolving mediators marks the dawn of resolution physiology and pharmacology. Mol. Aspects Med. 58:1-11 (2017).

28. Serhan, C. N. Treating inflammation and infection in the 21 st century: new hints from decoding resolution mediators and mechanisms. FASEB J. 31, 1273-1288 (2017).

29. Jin, Y. et al. Anti-angiogenesis effect of the novel anti-inflammatory and proresolving lipid mediators. Invest. Ophthalmol. Vis. Sci. 50, 4743-4752 (2009).

30. Hua, J. et al. The resolvin D1 analogue controls maturation of dendritic cells and suppresses alloimmunity in corneal transplantation. Invest. Ophthalmol. Vis. Sci. 55, 5944-5951 (2014)

31. English, J. T., Norris, P. C., Hodges, R. R., Dartt, D. A. \& Serhan, C. N. Identification and profiling of specialized pro-resolving mediators in human tears by lipid mediator metabolomics. Prostaglandins Leukot. Essent. Fatty Acids 117, 17-27 (2017).

32. Smith, R. E. et al. Secondary allergic T cell responses are regulated by dendritic cell-derived thrombospondin-1 in the setting of allergic eye disease. J. Leukoc. Biol. 100, 371-380 (2016)

33. Schlereth, S., Lee, H. S., Khandelwal, P. \& Saban, D. R. Blocking CCR7 at the ocular surface impairs the pathogenic contribution of dendritic cells in allergic conjunctivitis. Am. J. Pathol. 180, 2351-2360 (2012).

34. Kunert, K. S., Keane-Myers, A. M., Spurr-Michaud, S., Tisdale, A. S. \& Gipson, I. K. Alteration in goblet cell numbers and mucin gene expression in a mouse model of allergic conjunctivitis. Invest. Ophthalmol. Vis. Sci. 42, 2483-2489 (2001).
35. Merayo-Lloves, J., Calonge, M. \& Foster, C. S. Experimental model of allergic conjunctivitis to ragweed in guinea pig. Curr. Eye Res. 14, 487-494 (1995).

36. Toda, I., Shimazaki, J. \& Tsubota, K. Dry eye with only decreased tear break-up time is sometimes associated with allergic conjunctivitis. Ophthalmology 102 302-309 (1995).

37. Chiurchiu, V. et al. Proresolving lipid mediators resolvinD1, resolvin D2, and maresin 1 are critical in modulating T cell responses. Sci. Transl. Med 8, 353ra111 (2016).

38. Chiang, N. \& Serhan, C. N. Structural elucidation and physiologic functions of specialized pro-resolving mediators and their receptors. Mol. Aspects Med. 58:114-129 (2017).

39. Farkouh, A., Frigo, P. \& Czejka, M. Systemic side effects of eye drops: a pharmacokinetic perspective. Clin. Ophthalmol. 10, 2433-2441 (2016).

40. Saban, D. R. The chemokine receptor CCR7 expressed by dendritic cells: a key player in corneal and ocular surface inflammation. Ocul. Surf. 12, 87-99 (2014).

41. Proud, D. et al. Inflammatory mediator release on conjunctival provocation of allergic subjects with allergen. J. Allergy Clin. Immunol. 85, 896-905 (1990).

42. Pelikan, Z. Mediator profiles in tears during the conjunctival response induced by allergic reaction in the nasal mucosa. Mol. Vis. 19, 1453-1470 (2013).

43. Slagle, W. S., Slagle, A. M. \& Brough, G. H. Mucus fishing syndrome: case report and new treatment option. Optometry 72, 634-640 (2001).

44. Vichyanond, P., Pacharn, P., Pleyer, U. \& Leonardi, A. Vernal keratoconjunctivitis: a severe allergic eye disease with remodeling changes. Pediatr. Allergy Immunol. 25, 314-322 (2014).

45. Hodges, R. R. \& Dartt, D. A. Tear film mucins: front line defenders of the ocular surface; comparison with airway and gastrointestinal tract mucins. Exp. Eye Res. 117, 62-78 (2013).

46. Evans, C. M. et al. The polymeric mucin Muc5ac is required for allergic airway hyperreactivity. Nat. Commun. 6, 6281 (2015).

47. Ono, S. J. \& Abelson, M. B. Allergic conjunctivitis: update on pathophysiology and prospects for future treatment. J. Allergy Clin. Immunol. 115, 118-122 (2005).

48. Takeyama, K. et al. Activation of epidermal growth factor receptors is responsible for mucin synthesis induced by cigarette smoke. Am. J. Physiol. Lung Cell. Mol. Physiol. 280, L165-L172 (2001).

49. Lee, H. M. et al. Epidermal growth factor receptor signaling mediates regranulation of rat nasal goblet cells. J. Allergy Clin. Immunol. 107, 1046-1050 (2001).

50. Dartt, D. A., Kessler, T. L., Chung, E. H. \& Zieske, J. D. Vasoactive intestinal peptidestimulated glycoconjugate secretion from conjunctival goblet cells. Exp. Eye Res. 63, 27-34 (1996)

51. Rios, J. D. et al. Immunolocalization of muscarinic and VIP receptor subtypes and their role in stimulating goblet cell secretion. Invest. Ophthalmol. Vis. Sci. 40, 1102-1111 (1999).

52. Hodges, R. R. et al. Lipoxin A4 activates ALX/FPR2 receptor to regulate conjunctival goblet cell secretion. Mucosal Immunol. 10, 46-57 (2017).

53. Norling, L. V., Dalli, J., Flower, R. J., Serhan, C. N. \& Perretti, M. Resolvin D1 limits polymorphonuclear leukocyte recruitment to inflammatory loci: receptordependent actions. Arterioscler. Thromb. Vasc. Biol. 32, 1970-1978 (2012).

54. Bielory, B. P., O'Brien, T. P. \& Bielory, L. Management of seasonal allergic conjunctivitis: guide to therapy. Acta Ophthalmol. (Copenh) 90, 399-407 (2012).

55. Sun, Y. P. et al. Resolvin D1 and its aspirin-triggered 17R epimer. Stereochemical assignments, anti-inflammatory properties, and enzymatic inactivation. J. Biol. Chem. 282, 9323-9334 (2007).

56. Serhan, C. N. et al. Resolvins: a family of bioactive products of omega-3 fatty acid transformation circuits initiated by aspirin treatment that counter proinflammation signals. J. Exp. Med. 196, 1025-1037 (2002).

57. Magone, M. T., Chan, C. C., Rizzo, L. V., Kozhich, A. T. \& Whitcup, S. M. A nove murine model of allergic conjunctivitis. Clin. Immunol. Immunopathol. 87, 75-84 (1998). 